\title{
Continuous extraction of phenol and cresols from advanced pyrolysis oils
}

\author{
Yaseen Elkasabi ${ }^{1}$ (D) Charles A. Mullen ${ }^{1}$. Akwasi A. Boateng ${ }^{1}$
}

Received: 23 October 2019 / Accepted: 29 January 2020 / Published online: 27 February 2020

(c) This is a U.S. Government work and not under copyright protection in the U.S.; foreign copyright protection may apply 2020

\begin{abstract}
Production of value-added chemicals will ultimately enable commercialization of biofuels. While bio-oils contain phenolic compounds, their separation has generally been difficult due to very wide product distributions. Advances in pyrolysis have produced oils with low oxygen and narrow product distributions that have made separation processes possible. We have demonstrated the continuous extraction of phenolic compounds from fast pyrolysis oils from switchgrass, produced using catalytic and/or tail-gas reactive pyrolysis, which enables distillation due to the absence of unstable oxygenated compounds. Having successfully distilled the bio-oil, the distillate was first subjected to batch extraction with $10 \mathrm{M}$ $\mathrm{KOH}$ in varying ratios, and the yields of phenol and cresols were measured. Extraction yields of $15-20 \mathrm{wt} \%$ produced extracts where the proportion of measured phenolics consisted of $>50 \%$ phenol (i.e. "phenol selectivity"), an increase from $36 \%$ in the starting distillates. Washing the distillates with sodium bicarbonate before phenolic extraction helped to reduce excessive acid contamination. Countercurrent continuous extraction experiments using a Vigreux column attained steady-state after only a few minutes, although the phenol selectivity continued to increase for the duration of most experiments. Extraction yields ranged from 15 to $40 \mathrm{wt} \%$.
\end{abstract}

Keywords Pyrolysis · Bio-oil · Separations · Extraction · Phenol

\section{Introduction}

Thermochemical production of liquid biofuels proceeds primarily through fast pyrolysis [1, 2] and liquefaction [3]. While various strides have been made towards improving their scalability [4], the economic feasibility will require some innovative means of increasing profitability through value-added chemicals. Because of the similarity of underlying processes such as catalytic cracking operations [5], fast pyrolysis of biomass has the potential to adapt towards the existing refinery infrastructure [6], which will thereby save substantial capital investments normally made in new turn-key refineries [7]. Despite this advantage, stronger economic drivers are needed to advance pyrolysis biorefineries. The relatively low price of fossil fuels necessitates further reduction of the minimum fuel selling price (MFSP) of pyrolysis-based biofuels which currently stands at $\$ 4.00-5.50 / \mathrm{gal}[8,9]$. This can be achieved by means of (1) higher throughput (2) reduced operating

\footnotetext{
Mention of trade names or commercial products in this publication is solely for the purpose of providing specific information and does not imply recommendation or endorsement by the U.S. Department of Agriculture. USDA is an equal opportunity provider and employer.
}

Electronic supplementary material The online version of this article (https://doi.org/10.1007/s42452-020-2134-4) contains
supplementary material, which is available to authorized users.

Yaseen Elkasabi, yaseen.elkasabi@usda.gov| ${ }^{1}$ Eastern Regional Research Center, Agricultural Research Service, U. S. Department of Agriculture, 600 E. Mermaid Lane, Wyndmoor, PA 19038, USA. 
costs, primarily through improving failed catalysts (3) production and sale of high-value co-products.

In the petrochemical industry, overall, only $15 \%$ of an oil barrel contributes towards non-fuel products such as chemicals and materials [10]. Despite this low fraction, non-fuel products comprise nearly half of the industry's profits. Hence, for most refineries, profitability relies on efficient production and sale of chemicals, a synergy with which pyrolysis biorefining can aligned. For example, oxygenated compounds like 1,3-propanediol and acrylic acid are key components and building blocks of many other materials and chemicals. They must be produced catalytically since oxygenated compounds are not present in petroleum, though renewable pathways for 1,3-propanediol and others have been developed [11]. For example the commercial production of phenol uses the cumene process, a multi-step synthesis that is highly expensive. Considering that biomass pyrolysis oils emanate from lignocellulosic feedstocks structured from phenolic monomers, bio-oils offer the potential to supplement biofuels produced in parallel with high-value phenolic compounds, provided that those compounds could be catalytically modified and/or isolated to specific targets. Hence, technologies for both catalytic production and separation of phenolics will aid in the long-term synergy of refineries towards bioprocessing.

Overall, bio-oil separations process development [12] has lagged relative to the extensive efforts in deoxygenation catalysis. Reasons for this discrepancy include (1) the highly oxygenated compounds in bio-oils (2) a very wide distribution of molecular weight and compound types (3) thermal instabilities prohibiting even mild temperature elevation [13] and (4) the lack of approaches towards selectivity in separations. For example the distillation of petroleum feedstocks proceeds at temperatures above $350{ }^{\circ} \mathrm{C}$, but whole bio-oils from conventional pyrolysis tend to repolymerize upon heating [14], thereby producing water and solid residues. When it comes to extraction technologies, previous literature documents limited advancements of separation, wherein separated fractions are classified based on the solvent used for extraction [15]. However, the separated fractions hold the same issues: a highly-oxygenated population with a very wide distribution. The concentrations of each compound in bio-oil (whole or extracted fractions) are too small to carry out meaningful extent of separation $(<2-3 \mathrm{wt} \%)$.

However, novel pyrolysis processes have allowed the production of bio-oils that are more amenable towards post-pyrolysis product separations. Catalytic fast pyrolysis (CFP) $[16,17]$ and tail-gas reactive pyrolysis (TGRP) $[18,19]$ effectively reduce the oxygen content of biooils, such that the four aforementioned issues are at least partially mitigated. The thermally unstable oxygenated functional groups allow for standard distillation to proceed to three- to fourfold greater yields, in both batch [20] and continuous [21] modes. Distillation also allows for the generation of a coke residue coproduct for production of calcined coke $[22,23]$. The reduction in oxygen also narrows the product distribution of bio-oilswith the major species being aromatic hydrocarbons and phenolics-thereby enabling a more meaningful separation to take place, based on extracting specific chemical species. Using a strong base, extraction can take place on the whole distillates [24], producing a hydrocarbon-only fraction and a phenolics fraction separately. While such a basic extraction process makes the refining into fuel hydrocarbons easier, improvements are needed to advance continuous isolation. Elucidating these extraction parameters will pave the way for continuous extraction of phenolic compounds. This paper establishes some principles in continuous extraction of phenol from bio-oils.

\section{Experimental}

\subsection{Fast pyrolysis}

Fast-pyrolysis of switchgrass was carried out in the Eastern Regional Research Center (ERRC) fluidized bed fast pyrolysis system, as described previously [19]. For TGRP experiments, a fraction of the non-condensable gas stream was mixed with $\mathrm{N}_{2}$ and recycled into the fluidized bed, using a preheater and gas blower. The pyrolysis system recycled the tail gas in the range of $50-70$ vol\%, with the balance consisting of nitrogen. The electrostatic precipitator (ESP) oil fraction served as the basis for all experiments.

\subsection{Bio-oil distillation}

Distillation and extraction proceeded according to previously-established protocols, with slight modification. Briefly, bio-oil was added to a quartz round-bottom flask (filled to less than one-third of the flask volume) and gradually heated with a heating mantle. Distillates were collected with a short-path distilling head and condenser. Both the overhead and bottom temperatures were recorded at the overhead vapor and bottoms flask, respectively. Temperatures were recorded at the end of each fraction collection. When the flask temperature reached $350^{\circ} \mathrm{C}$, flask heating was halted. Aqueous phases were decanted from any biphasic fractions, and all organic fractions were combined. 


\subsection{Extraction (batch and continuous)}

For batch experiments, $5 \mathrm{~mL}$ of bio-oil distillates and the appropriate amount of $10 \mathrm{M} \mathrm{KOH}$ (aq) were added into centrifuge tubes in predetermined ratios. After vigorous mixing and vortexing, the tubes were centrifuged, and the two phases were separated. The $\mathrm{NaOH}$ phase was acidified with concentrated $\mathrm{HCl}$ dropwise, until a clear water-phase precipitated. For continuous experiments, a Vigreux column was equipped vertically to form an updraft countercurrent extraction setup (see Fig. 1 inset). The top of the column was fitted with a Luer vacuum adapter, whereby concentrated $\mathrm{NaOH}(\mathrm{aq})$ was injected downdraft. The bottom of the column was fitted with an airfree Schlenk adapter, custom-made with a syringe port for bio-oil injection to be introduced via HPLC pump. $\mathrm{NaOH}$ was introduced at $1.2 \mathrm{~mL} / \mathrm{min}$, and bio-oil distillates were introduced at either $4.0 \mathrm{~mL} / \mathrm{min}$ (Condition 1) or $2.0 \mathrm{~mL} / \mathrm{min}$ (Condition 2). Samples were collected approximately every $5 \mathrm{~min}$. $\mathrm{NaOH}$ phases were acidified dropwise in the same manner as batch experiments to produce phenolics.

\subsection{Characterization}

Elemental analysis (CHNS) was conducted via a Thermo EA1112 CHNS analyzer, and results were verified by analysis from an outside party (Robertson Microlit Laboratories,
Ledgewood, NJ, USA). Oxygen content was calculated by difference, and water content was used to subtract $\mathrm{H}$ and $\mathrm{O}$ and recalculate results on a dry basis. Moisture content was measured with Karl-Fischer titration in methanol with Hydranal Karl-Fischer Composite 5 (Fluka) as the titrant. Total acid number (TAN) was measured using a Mettler T70 autotitrator using $0.1 \mathrm{M} \mathrm{KOH}$ in isopropanol as the titrant and wet ethanol as the titration solvent. Gas chromatography with mass spectroscopy (GC-MS) analysis of liquid products was performed on a Shimadzu GCMS QC-2010. The column used was a DB-1701, $60 \mathrm{~m} \times 0.25 \mathrm{~mm}, 0.25 \mu \mathrm{m}$ film thickness. The oven temperature was programmed to hold at $45^{\circ} \mathrm{C}$ for $4 \mathrm{~min}$, ramp at $3^{\circ} \mathrm{C} \mathrm{min}-1$ to $280^{\circ} \mathrm{C}$ and hold at $280^{\circ} \mathrm{C}$ for $20 \mathrm{~min}$. The injector temperature was $250^{\circ} \mathrm{C}$, and the injector split ratio set to $30: 1$. Helium carrier gas flowed at $1 \mathrm{~mL} \mathrm{~min}^{-1}$. NMR spectrometry of phenolic extracts proceeded according to previously established protocols [25].

\section{Results and discussion}

\subsection{Batch extraction}

Figure 1 shows the logic flow model for the separation of advanced pyrolysis oils into phenolics and hydrocarbon streams. The overall goal for post-pyrolysis refining was

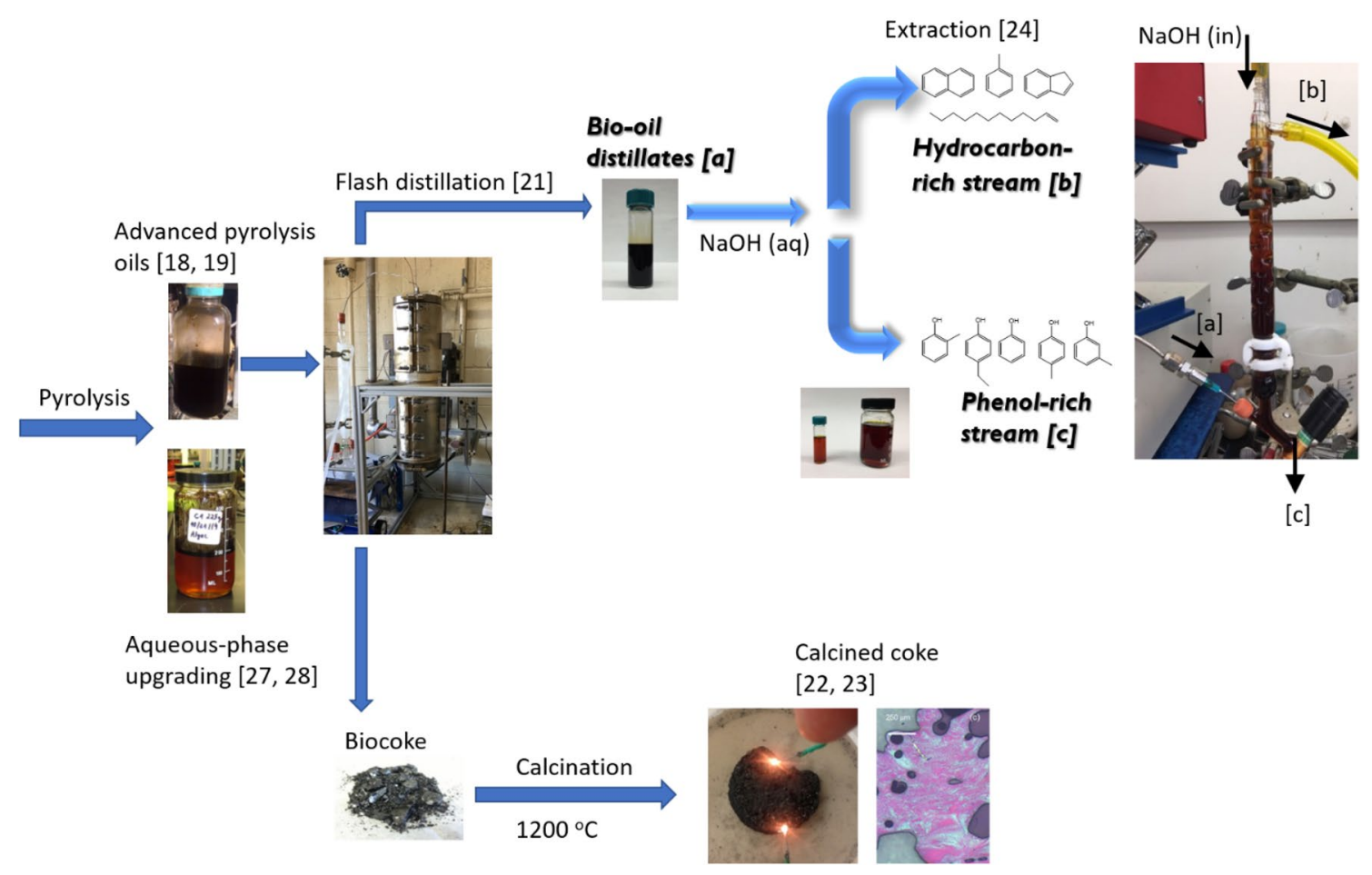

Fig. 1 Flow chart for refining bio-oils from advanced pyrolysis processes 
to maximize product utilization while making minimal use of costly processing steps involving pressurized vessels. This takes advantage of the fact that when flash distillation is operated semi-continuously, it can produce the distillates needed for continuous production of phenolics and hydrocarbons, with the coke residue accumulated and subsequently collected from the drum during alternate semi-continuous processing with a second drum. With this process the hydrocarbon stream can undergo hydrogenation for production of fuel grade hydrocarbons. One advantage of this arrangement is that the coke residues could be a source of added revenue with the potential to reduce MFSP, due to its relatively high purity compared with fossil-derived coke [9]. During the phenolic extraction step, most of the acidic compounds are sequestered into a separate aqueous phase [24], though a minimal amount can remain $(\sim 1 \%)$ in the hydrocarbon phase. Phenolics could either be purified into phenol and/or undergo HDO (hydrodeoxygenation).

In TGRP/CFP oils, the primary phenol compounds detected with GC-MS are: phenol, cresols $(o-, m-, p-)$, and 4-ethylphenol. Phenol can be concentrated with fractional distillation, though this method alone can eventually become difficult, due to its very similar volatility to the cresols. While extraction with excess concentrated $\mathrm{NaOH}$ can typically remove all the phenolics together, the oil:base ratio will determine the extent of phenol removal. Owing to the lack of substituted electron donating alkyl groups, the $\mathrm{pK}_{\mathrm{a}}$ of phenol (9.9) is slightly lower than that of the other alkyl phenolics ( $>10)$ present. Also considering that the concentration of phenol present is the greatest, relative to that of cresol/ethylphenol, one could expect an increase in phenol concentration post-extraction, though the extent of its increase would depend on the associated yield.

We performed batch extraction experiments as a way to guide subsequent continuous separation experiments. Table 1 displays the compositional profiles of both the bio-oil distillates used and the extract/raffinate fractions from the batch process. Phenol selectivity is defined as the concentration of phenol divided by the total concentration of phenols measured. Generally, as the oil:base ratio decreases, the phenol selectivity decreases. At the same time, two things occur, depending on the initial oil:base ratio (1) the first phenol selectivity percentage is of significantly greater value than that of the starting distillates. (2) The total yield of extract using the specified oil:base ratio increases, starting from a relatively low value of 2.6 $w t \%$. Overall, the total measured concentration of phenol remains relatively unchanged, wavering between 11 and $12 \mathrm{wt} \%$. To improve the yield of phenol, a ratio between 3.8 and 3.1 would be appropriate for further testing in continuous experiments, as it would compromise between a high phenol selectivity and a higher yield. This choice would also provide phenol(s) that contain less non-phenolic contaminants, as higher yield extraction ratios (e.g. with $48 \mathrm{wt} \%$ yield) increase the probability of removal of some hydrocarbons like BTEX. Similar trends can be seen when the extraction is performed on bio-oil distillates of relatively low phenolics content (supplementary information Tables S1 and S2), when the extraction is repeated on the same sample in sequence. However, low phenolics
Table 1 Compositions and yields for batch extraction of phenolics from advanced pyrolysis bio-oil distillates from switchgrass

\begin{tabular}{llllllll}
\hline & Distillates & Extract & & \multicolumn{5}{l}{ Raffinate } \\
\hline Distillates:NaOH (vol) & - & $3.8: 1$ & $3.1: 1$ & $2.5: 1$ & $3.8: 1$ & $3.1: 1$ & $2.5: 1$ \\
Extraction yield (\%) & - & 2.6 & 16.2 & 48 & - & - & - \\
Phenolic yield (\%) & & 2.9 & 25.3 & 81 & & & \\
BTEX (wt\%) & & 0.97 & 2.48 & 3.93 & 7.68 & 8.81 & 14.08 \\
Trimethylbenzene & $<0.01$ & 0.16 & 0.15 & 0.46 & 0.98 & 1.15 & 1.86 \\
Phenols & 16.56 & 18.29 & 25.87 & 27.91 & 16.61 & 13.57 & 7.31 \\
Phenol & 5.99 & 10.68 & 12.01 & 10.62 & 5.85 & 4.26 & 1.36 \\
o-cresol & 2.43 & 1.83 & 3.02 & 3.45 & 2.53 & 2.26 & 1.64 \\
p-cresol & 2.1 & 1.71 & 3.03 & 3.52 & 2.16 & 1.78 & 0.78 \\
m-cresol & 2.33 & 2.11 & 3.41 & 3.75 & 2.32 & 2.00 & 0.91 \\
2,4-Dimethylphenol & 0.02 & 0.04 & 0.08 & 0.89 & 0.10 & 0.09 & 1.02 \\
4-Ethyl phenol & 3.69 & 1.94 & 4.33 & 5.69 & 3.66 & 3.20 & 1.61 \\
Methoxyphenols & 0.92 & 0.65 & 1.37 & 1.55 & 0.90 & 0.62 & 0.20 \\
Naphthalenes & 8.44 & 1.63 & 3.11 & 4.49 & 9.05 & 10.46 & 16.45 \\
Olefins & 3.19 & 0.53 & 1.19 & 1.79 & 3.40 & 3.92 & 6.21 \\
Acetic acid & 1.81 & 7.89 & 1.50 & 0.53 & $<0.01$ & 0.02 & $<0.01$ \\
"Phenol selectivity" & 36 & 58 & 46 & 38 & 35 & 31 & 19 \\
\hline
\end{tabular}

${ }^{a}$ Benzene, toluene, ethylbenzene, xylenes 
concentration in the distillates further hinders the ability to have both significant yields and higher selectivities.

We also observed that the concentration of acetic acid in the lowest oil:base ratio extract was just under $8.0 \mathrm{wt} \%$, nearly 4 times that of the starting distillates. This naturally arises from the ability of sodium hydroxide to remove both strong and weak acid groups. Although the acid-extracted distillates contain little acetic acid, this amount will get collected and concentrated into the phenolic extract, due to the strength of $\mathrm{NaOH}$ towards removing both phenolics and carboxylic acids ( $\mathrm{pK}_{\mathrm{a}} \sim 4.8$ ). As a way to avoid transfer of acid groups to the final extract, we also performed a set of extractions with sodium bicarbonate as a pre-extraction step to selectively remove acid groups. Table 2 shows the characterization of the distillates post-acid extraction as well as the extract products. Based on the larger oil:base ratio used, the extract was of a greater yield for the phenol selectivity obtained, relative to the results in Table 1. Also, the acetic acid concentration of the extract was significantly less than that of the extract in Table 1.

\subsection{NMR characterization}

The GC-MS data accounts only for those compounds that are within the measurable range and, specifically for quantitative results, those finite compounds for which measurements have been calibrated. To ensure that the isolation of phenol is concentrated from all phenolics (and the whole fraction in general), we performed NMR spectrometry on

Table 2 Phenolic extraction yields from bio-oil distillates, using extraction with $\mathrm{Na}_{2} \mathrm{CO}_{3}$ to remove carboxylic acids as a pre-step (4.5:1 oil:base ratio)

\begin{tabular}{llll}
\hline & $\begin{array}{l}\text { Distillates } \\
\text { (acid- } \\
\text { extracted) }\end{array}$ & Extract (4.4:1) & Raffinate (4.4:1) \\
\hline Extraction yield (\%) & - & 8.2 & - \\
Phenolic yield (\%) & & 10.1 & \\
BTEX & 6.83 & 1.57 & 6.87 \\
Trimethylbenzene & 1 & 0.23 & 0.9 \\
Phenols & 18.08 & 23.26 & 15.5 \\
Phenol & 6.63 & 12.31 & 5.42 \\
O-cresol & 2.67 & 2.46 & 2.3 \\
P-cresol & 2.23 & 2.39 & 1.93 \\
M-cresol & 2.54 & 2.95 & 2.34 \\
2,4-Dimethylphenol & 0.01 & 0.01 & $<0.01$ \\
4-Ethyl phenol & 4 & 3.14 & 3.51 \\
Methoxyphenols & 0.96 & 1.1 & 0.7 \\
Naphthalenes & 9.46 & 2.34 & 8.76 \\
Olefins & 3.5 & 0.85 & 3.12 \\
Acetic acid & $<0.01$ & 1.56 & $<0.01$ \\
"Phenol selectivity" & 37 & 53 & 35 \\
\hline
\end{tabular}

the phenolic extracts of Table 1 . Figure 2 shows the ${ }^{1} \mathrm{H}$ NMR associated with the extracts. In the spectra, peaks between 6.8-8.0 ppm represent the aromatic ring protons, peaks from 2.1-2.3 are alkyl groups directly attached to the ring, while the peaks at 1.0-1.5 are alkyl groups further separated from the aromatic ring (e.g. the methyl group of ethyl phenol). The specific resonances for phenol are noted on the blow up of the aromatic region and are seen at $7.15,6.95$ and 6.78 for the protons at the meta, para and ortho positions to the hydroxyl group, respectively. The decrease in overlap in the resonance signals for phenol and other phenolics for the 3.8/1 extract can be also seen in the aromatic region blowup. The extract with the highest concentration of phenol contains the highest ratio of peaks associated with aromatic hydrogen to the peaks for alkyl groups. The ratio of the peak areas for the meta protons on phenol (triplet at $7.15 \mathrm{ppm}$ ) to the resonances between 3.1 and $3.3 \mathrm{ppm}$ are $0.44 / 1,0.28 / 1$ and $0.22 / 1$ for the extractions performed at the 3.8:1, 3.1:1 and 2.5:1 ratios, respectively. This may indicate not only that the phenol/alkyl phenol ratio has increased but also that the ratio of other non-alkyl substituted phenolics (catechols or napthalenols) to alkyl phenols has also increased.

\subsection{Continuous extraction}

Based on the batch extraction data collected, we chose two oil:base ratios (3.3:1 and 1.6) for testing in a continuous flow system. 3.3:1 was chosen as a compromise between the higher phenol selectivity of the 3.8:1 extract and the higher yield of the 3.1:1 extract. 1.6:1 was chosen as an evaluation point for the removal of most phenolic compounds, rather than for the selective removal of phenol. A Vigreux column provides extra surface area in order to break apart any emulsions, as well as to facilitate mixing of both phases. Despite the higher density of sodium phenoxides, their viscous nature necessitates that extra time and distance are allowed for settling from the distillates, so injection of the distillates occurs at the top of the Schlenk adapter through a custom-made port. Based on initial flow tests with simulated pyrolysis oil distillates [26] (data not shown), flow rates of $\mathrm{NaOH}$ greater than $1.0 \mathrm{~mL} /$ min are needed to ensure adequate penetration of flow downward, as well as to ensure that any phenolic salts formed do not deposit or suspend in the updraft phase.

Total mass balance closures for all runs (data not shown) averaged close to $90 \mathrm{wt} \%$, based upon the summation of all samples obtained in a run, as well as liquid remaining in the column. Some reasons for the remaining $10 \%$ loss emanate from sample transfer between vials and loss of organics to aqueous phases upon acidification of the phenolic salts. For continuous experiments on both flow ratios, Fig. 3 shows the time-on-stream mass yields for both 


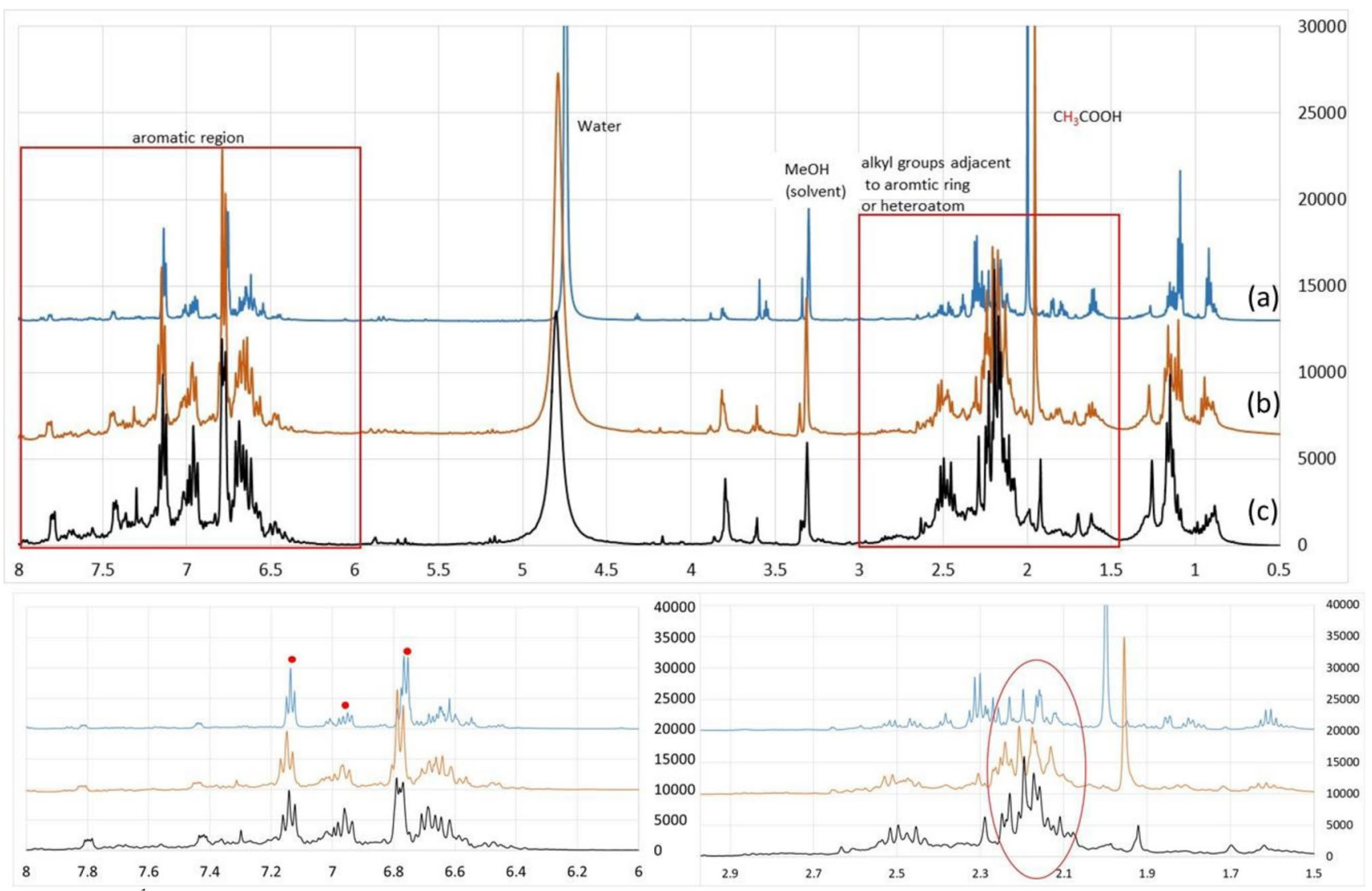

Fig. $2{ }^{1} \mathrm{H}$ NMR spectra of isolated phenolics using a 3.8:1, b 3.1:1, c 2.5:1 extraction ratios. Greater concentrations of phenol are isolated with higher ratios. Full plot scaled to methanol solvent peak. Blow up plots scaled to meta protons peak for phenol $(t, 7.15)$.
Boxes on main plot indicate area of blow ups. Red dots denote phenol resonances. Circle denotes resonances for alkyl groups attached to aromatic ring

length (and by extension, the extraction time during continuous flow), the extra degree of mixing allowed for batch experiments, and the volumetric effects in continuous experimentation. Due to the widely varied effects these parameters can have on final product distribution, a more thorough modeling approach can aide with increasing the efficiency of continuous extraction. The results illustrate the feasibility of continuous phenol extraction, though modification of process parameters (primarily the column length) is required for further improvement.

Comparisons of phenolic selectivity for continuous experiments, when made in conjunction with the total phenolics in the samples, give insight into the compositional convergence. Figure 5 shows dual plots for each run: overlay of the TOS phenol selectivity with the percent phenolics present relative to all measured compounds. In contrast to the other trends illustrated, the phenol selectivity increases more gradually over the course of the experiment for 3.3:1 flow rate, with a true steady state value not fully realized. Simultaneously, the total percentage of phenolics across each sample did not change. Hence, while the 

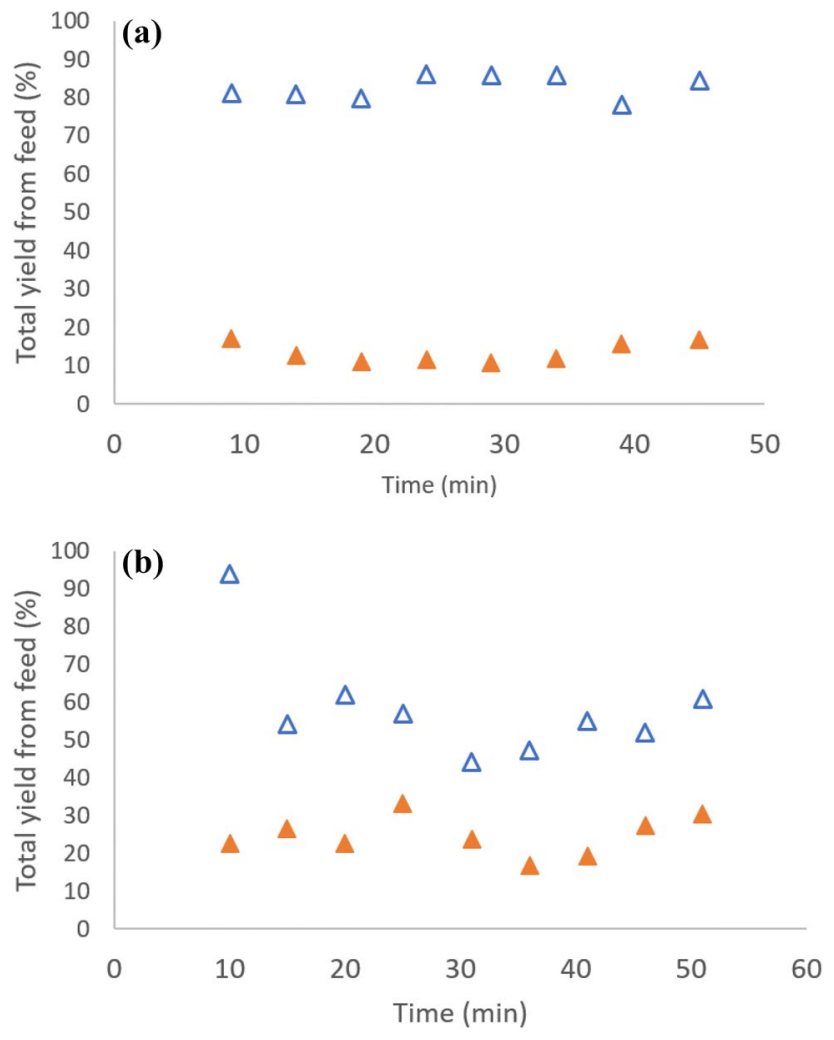

Fig. 3 TOS mass yields for a condition 1 (3.3:1 flow ratio) and $\mathbf{b}$ condition 2 (1.7:1 flow ratio), based on extract (filled triangles) and raffinate (empty triangles)

total flow rates converged quickly towards a mass steady state, the composition continuously changed and concentrated the phenol to at least $42 \mathrm{wt} \%$, indicating that countercurrent extraction can effectively remove phenol from pyrolysis oils. One possible configuration for biorefining is to extract this amount continuously while the raffinate undergoes HDO, such that a biorefinery can coproduce both fuels and coproducts. Conceivably, this extracted stream would require further distillation to purify the phenol, but increased phenol selectivity would save capital and operating costs by shortening the required column height/diameter.

The most direct way to compare effectiveness between runs (continuous and/or batch) is to compare the relative changes in both the mass yields and phenolic selectivities. Simultaneous comparison amongst all runs can best be illustrated by plotting each pair of values (yield vs. selectivity) for all runs (Fig. 6). Data from this plot also includes extraction data on lower oxygenated oil distillates in the supplemental section. Figure 6 immediately makes apparent that all the data points fall on a trend curve, suggesting that for all extractions on whole distillates, prediction of selectivity relates primarily to the extraction yield more so than to the exact chemical composition of the distillates.
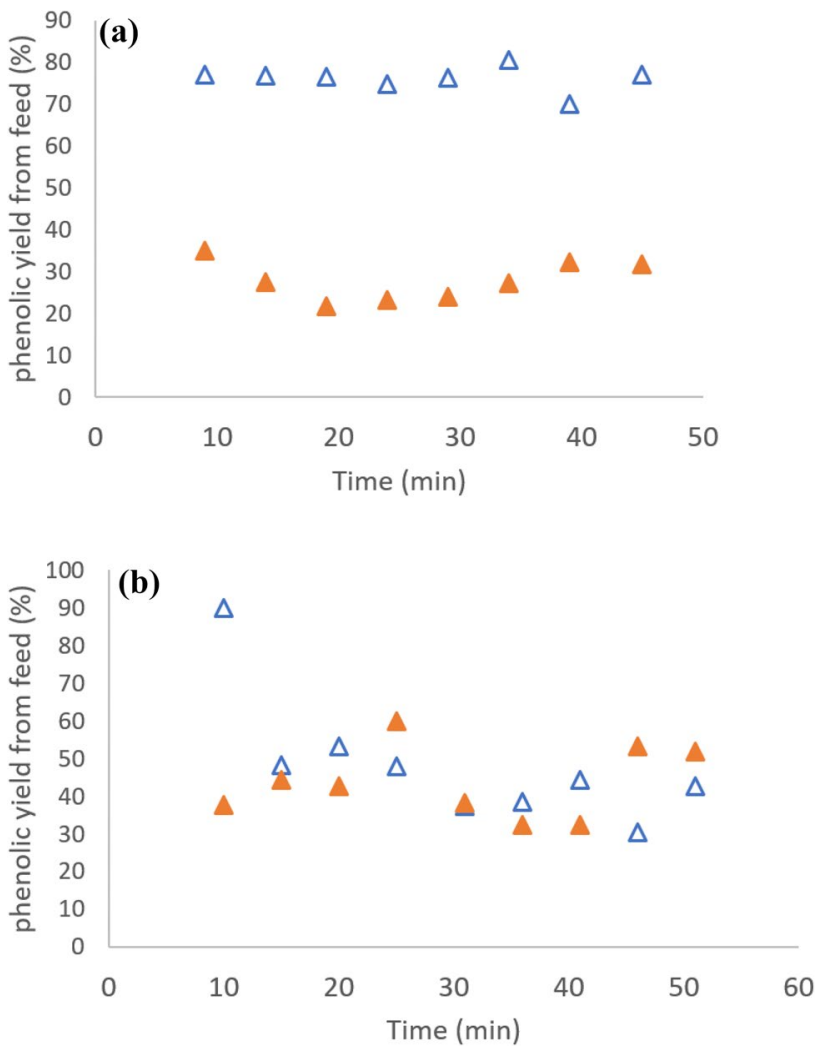

Fig. 4 TOS phenolic yields for a condition 1 (3.3:1 flow ratio) and $\mathbf{b}$ condition 2 (1.7:1 flow ratio), based on extract (filled triangles) and raffinate (empty triangles)
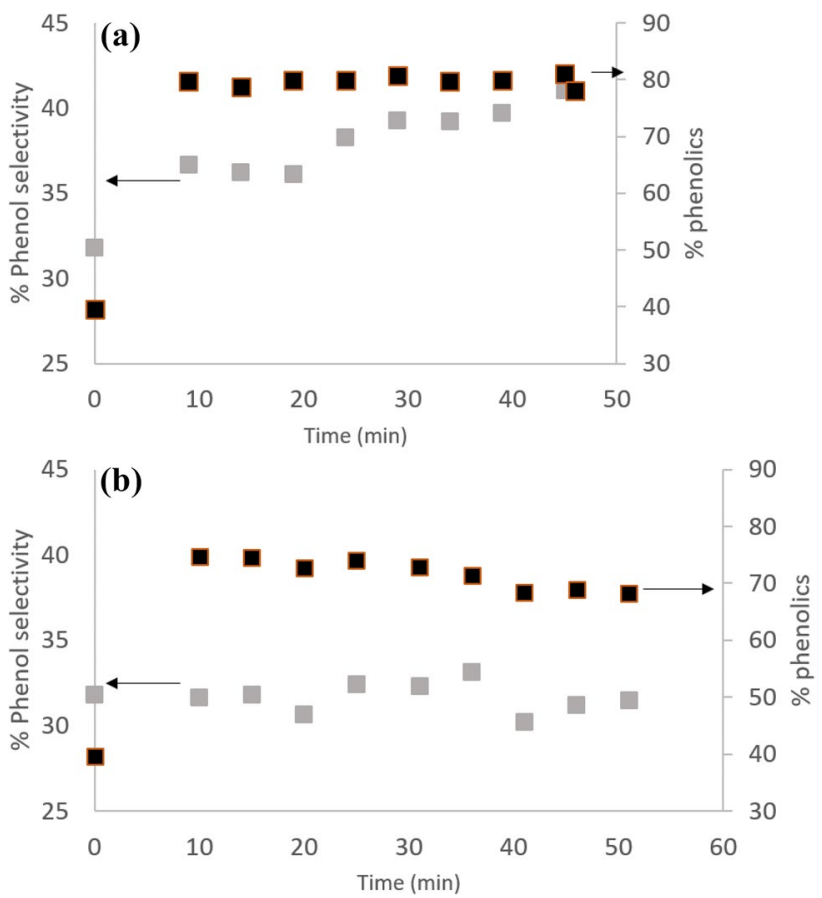

Fig. 5 TOS phenol selectivity and total percent phenolics of the extract phase for $\mathbf{a}$ condition 1 and $\mathbf{b}$ condition 2 . Inset arrows indicate proper axes 


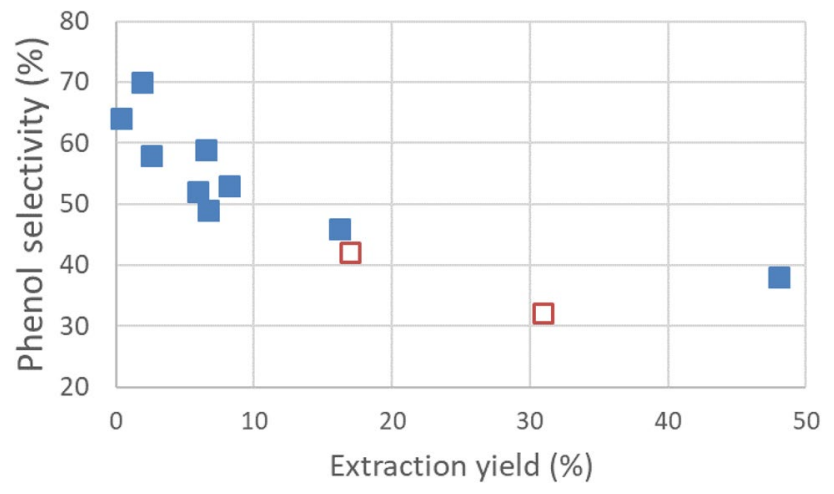

Fig. 6 Phenol selectivity with respect to extract yields, for batch phenolic extraction experiments. (Filled: batch extraction. Open: steady-state continuous extraction)

Theoretically, continuous decrease of yield towards 0 will approach $100 \%$ selectivity, while complete removal of phenolics must produce selectivity equal to that of the starting distillates (i.e. $32 \mathrm{wt} \%$ ). Thus, the trend follows a non-linear decay curve trend. Although the lower oxygen oils have significantly smaller concentrations of phenolics, the initial fraction of phenolics comprising of phenol is nonetheless the same ( 28 wt $\%)$, which likely contributes to the near-universal trend of Fig. 6. While Fig. 6 indicates that selective extraction of phenol is unlikely to be economical by itself, it can serve as a guide for economically optimizing production of phenol versus hydrocarbon fuels.

\section{Conclusions}

Using advanced pyrolysis bio-oil distillates, we demonstrated the continuous extraction of phenolics under countercurrent flow conditions. Mass flow steady-state was achieved under relatively short times, while concentrations of phenol gradually increased in concentration for the duration of the runs. While higher oil:base ratios resulted in the transfer of acidic groups, pre-extraction with sodium bicarbonate successfully removed the acetic acid selectively. For incorporation with continuous unit operations, further optimization of the column configurations will be required to increase efficiency of operation.

Acknowledgements The authors acknowledge the help of Thomas Coleman and Craig Einfeldt for assistance with the bench-scale pyrolysis experiments and Lucas Raymundo for operation of the bench-scale pyrolysis system.

\section{Compliance with ethical standards}

Conflict of interest On behalf of all authors, the corresponding author states that there is no conflict of interest.

\section{References}

1. Chen D, Yin L, Wang H, He P (2014) Pyrolysis technologies for municipal solid waste: a review. Waste Manag 34:2466-2486

2. Bridgwater $A$ (2018) Challenges and opportunities in fast pyrolysis of biomass: oart II. Johnson Matthey Tech Rev 62:150-160

3. Tian C, Li B, Liu Z, Zhang Y, Lu H (2014) Hydrothermal liquefaction for algal biorefinery: a critical review. Renew Sustain Ener Rev 38:933-950

4. Sharifzadeh M, Sadeqzadeh M, Guo M, Borhani T, Murthy Konda N, Garcia M, Wang L, Hallett J, Shah N (2019) The multi-scale challenges of biomass fast pyrolysis and bio-oil upgrading: review of the state of art and future research directions. Prog Enery Combust Sci 71:1-80

5. Olsen T (2014) An oil refinery walk-through. Chem Eng Pract 110:34-40

6. Talmadge MS, Baldwin RM, Biddy MJ, McCormick RL, Beckham GT, Ferguson GA, Czernik S, Magrini-Bair KA, Foust TD, Metelski PD, Hetrick C, Nimlos MR (2014) A perspective on oxygenated species in the refinery integration of pyrolysis oil. Green Chem 16:407-453

7. Speight JG (1999) The chemistry and technology of petroleum, 3rd edn. Marcel Dekker, New York, pp 215-216

8. Brown T, Brown R (2013) Techno-economics of advanced biofuels pathways. RSC Adv 3:5758-5764

9. Sorunmu Y, Billen P, Elkasabi Y, Mullen C, Macken N, Boateng A, Spatari $S$ (2017) Fuels and chemicals from equine-waste-derived tail gas reactive pyrolysis oil: technoeconomic analysis, environmental and exergetic life cycle assessment. ACS Sustain Chem Eng 5:8804-8814

10. Turcan C (2017) Source of over half Exxon's income, the significant of petrochemicals. Seeking Alpha. https://seekingalp ha.com/article/4080896-source-half-exxons-income-significan ce-petrochemicals

11. Zeng A (2019) New bioproduction systems for chemicals and fuels: needs and new development. Biotechnol Adv 37:508-518

12. Pinheiro Pires A, Arauzo J, Fonts I, Domine M, Fernandez Arroyo A, Garcia-Perez M, Montoya J, Chejne F, Pfromm P, Garcia-Perez $M$ (2019) Challenges and opportunities for bio-oil refining: a review. Energy Fuels 33:4683-4720

13. Oasmaa A, Fonts I, Pelaez-Samaniego M, Garcia-Perez M, GarciaPerez M (2016) Pyrolysis oil multiphase behavior and phase stability: a review. Energy Fuels 30:6179-6200

14. Oasmaa A, Kuoppala E (2008) Solvent fractionation method with brix for rapid characterization of wood fast pyrolysis liquids. Energy Fuels 22:4245-4248

15. Lu D, Liu Y, Wang D, Ye Y (2013) Separation of chemicals from bio-oil and their application prospects. Chem Ind For Prod 33:137-143

16. Liu C, Wang H, Karim AM, Sun J, Wang Y (2014) Catalytic fast pyrolysis of lignocellulosic biomass. Chem Soc Rev 43:7594-7623

17. Serrano-Ruiz J, Dumesic J (2011) Catalytic routes for the conversion of biomass into liquid hydrocarbon transportation fuels. Energy Environ Sci 4:83-99

18. Mullen CA, Boateng AA, Goldberg NM (2013) Production of deoxygenated biomass fast pyrolysis oils via product gas recycling. Energy Fuels 27:3867-3874

19. Raymundo L, Mullen C, Strahan G, Boateng A, Trierweiler J (2019) Deoxygenation of biomass pyrolysis vapors via in situ and ex situ thermal and biochar promoted upgrading. Energy Fuels 33:2197-2207

20. Elkasabi Y, Mullen CA, Boateng AA (2014) Distillation and isolation of commodity chemicals from bio-oil made by tail-gas reactive pyrolysis. ACS Sustain Chem Egr 2:2042-2052 
21. Elkasabi Y, Mullen CA, Boateng AA, Brown A, Timko MT (2019) Flash distillation of bio-oils for simultaneous production of hydrocarbons and green coke. Ind Eng Chem Res 58:1794-1802

22. Elkasabi $Y$, Boateng AA, Jackson MA (2015) Upgrading of bio-oil distillation bottoms into biorenewable calcined coke. Biomass Bioenergy 81:415-423

23. Elkasabi Y, Darmstadt H, Boateng AA (2018) Renewable biomass-derived coke with texture suitable for aluminum smelting anodes. ACS Sustain Chem Eng 6:13324-13331

24. Elkasabi Y, Mullen CA, Boateng AA (2015) Aqueous extractive upgrading of bio-oils created by tail-gas reactive pyrolysis to produce hydrocarbons and phenols. ACS Sustain Chem Eng 3:2809-2816

25. Elkasabi Y, Liu Q, Choi YS, Strahan G, Boateng AA, Regalbuto JR (2017) Bio-oil hydrodeoxygenation catalysts produced using strong electrostatic adsorption. Fuel 207:510-521

26. McVey M, Elkasabi Y, Ciolkosz D (2019) Separation of BTX chemicals from biomass pyrolysis oils via continuous flash distillation.
Biomass Convers Biorefin. https://doi.org/10.1007/s13399-01900409-1

27. Satinover SJ, Elkasabi Y, Nuñez A, Rodriguez M Jr, Borole AP (2019) Microbial electrolysis using aqueous fractions derived from tail-gas recycle pyrolysis of willow and guayule. Bioresour Technol 274:302-312

28. Davidson SD, Lopez-Ruiz JA, Flake M, Cooper AR, Elkasabi Y, Morgano MT, Dagle VL, Albrecht KO, Dagle RA (2019) Cleanup and conversion of biomass liquefaction aqueous phase to $C_{3}-C_{5}$ olefins over $\mathrm{Zn}_{\mathrm{x}} \mathrm{Zr}_{\mathrm{y}} \mathrm{O}_{\mathrm{z}}$ catalyst. Catalysts 9:923-937

Publisher's Note Springer Nature remains neutral with regard to jurisdictional claims in published maps and institutional affiliations. 